\title{
Lung adenocarcinoma patient progression with gastrointestinal metastasis response to subsequent tyrosine kinase inhibitors (TKIs) from re-biopsy of new occurring driver gene mutation
}

\author{
Chunhua Zhou", Binjie Yan", Liang Zeng", Yi Xiong, Li Liu, Nong Yang, Yongchang Zhang \\ Department of Medical Oncology, Lung Cancer and the Gastrointestinal Unit, Hunan Cancer Hospital/The Affiliated Cancer Hospital of Xiangya \\ School of Medicine, Changsha 410013, China \\ "These authors contributed equally to this work. \\ Correspondence to: Yongchang Zhang, MD; Nong Yang, MS. Department of Medical Oncology, Lung Cancer and the Gastrointestinal Unit, Hunan \\ Cancer Hospital/The Affiliated Cancer Hospital of Xiangya School of Medicine, Changsha 410013, China. \\ Email: zhangyongchang@csu.edu.cn; yangnong0217@163.com.
}

Submitted Mar 09, 2018. Accepted for publication Jul 10, 2018.

doi: $10.21037 /$ jtd.2018.07.97

View this article at: http://dx.doi.org/10.21037/jtd.2018.07.97

\section{Introduction}

Primary lung cancer frequently metastasizes to the lung, lymph nodes, brain, liver, adrenal glands and bones (1). The clinical incidence of gastrointestinal (GI) metastasis from lung cancer has been reported to be as low as $0.2-1.7 \%$ (2). Studies might be difficult to organize because the clinical incidence and associated mortality rates are low and due to its lack of symptoms; thus, true understanding of the clinical presentation of metastases of primary lung cancer to the GI tract is currently incomplete (3). Due to a lack of pathology and molecular detection, lung cancer with GI metastasis is reported to have a poor prognosis with a mean survival of only 4-8 weeks (4).

In this study, we will describe three $E G F R$-mutation positive and $A L K$-negative lung adenocarcinoma patients with metastasis to the GI tract. The newly occurring drive gene mutations were detected by re-biopsy and next generation sequencing (NGS). Follow-up data showed that patients could benefit from subsequent target therapy.

\section{Cases presentation}

\section{Patient 1}

A 58-year-old non-smoking woman came to our hospital November 1, 2012 and received surgery for lumps in left lung. She was diagnosed with pT2aN2M0 (stage IIIa) with lung adenocarcinoma, $E G F R$ wild-type and $A L K$ negative, detected by amplification refractory mutation system (ARMS). The patient received 4 cycles of adjuvant chemotherapy. PET-CT showed metastasis to the lungs and left pleural 14 months later. Magnetic resonance imaging (MRI) showed bone metastases in the left femoral head, confirming progression of disease (PD). Treatment was initiated with pemetrexed combined with cisplatin; 4 cycles were given. After first-line chemotherapy, a liquid biopsy using NGS (Burning Rock, Guangzhou, China), including EGFR, ALK, ROS1, MET, RET, BRAF, ERBB2 and KRAS, was performed (Figure 1). However, all of the 8 driver genes were negative (Figure $1 B$ ); single pemetrexed was added as maintenance treatment for 12 months. Palliative radiotherapy was given to the patient for bone metastases. Partial response (PR) was evaluated by computed tomography (CT) for a total of 19 months. Newly occurring rectal metastasis were confirmed by CT and enteroscopy, and the patient was diagnosed with PD (Figure 1C, upper panel). Re-biopsy from the rectal metastasis was evaluated as poorly differentiated lung adenocarcinoma (Figure 1C, middle panel). Immunohistochemistry (IHC) was CK, EMA, CK7, TTF-1 and Napsin A was positive. CK20, CDX-2, Syn, Cga, CD3 and CD20 were negative (Figure 1C, lower panel). Ki67 was $30 \%$. The genotype was accessed using a CD74-ROS1 rearrangement like the newly occurring diver gene mutations detected by NGS 
A Timeline and duration of each treatment Surgery

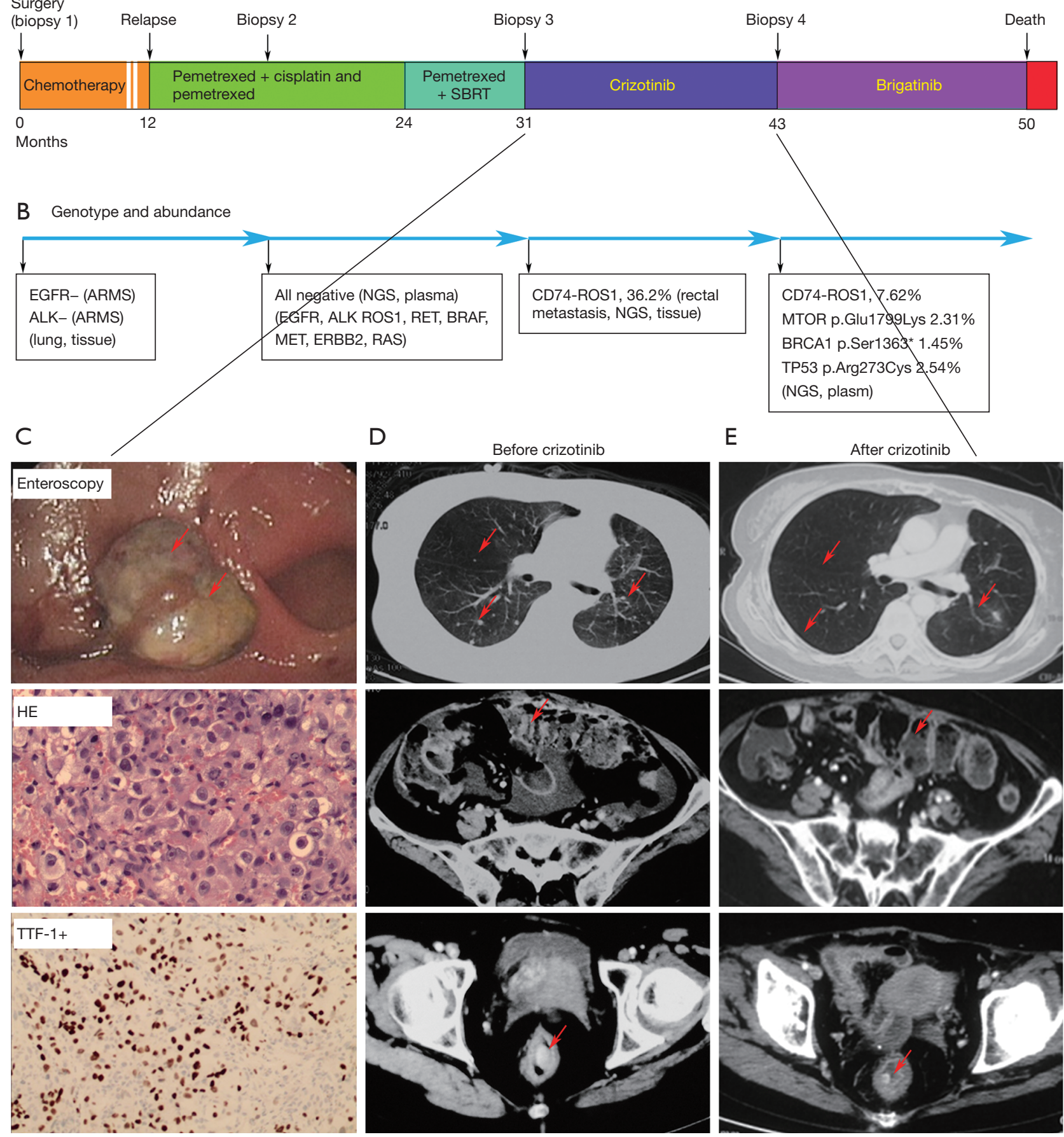

Figure 1 Lung adenocarcinoma patient progression with gastrointestinal metastasis benefited from re-biopsy and crizotinib. Panel A shows the various treatments of the lung adenocarcinoma patient, as well as the duration of each treatment; panel B shows the genotypes and the abundance of mutations detected by NGS for the lung adenocarcinoma patient under the various treatments; panel C (upper) shows the newly occurring rectal metastasis, confirmed by enteroscopy; panel C (middle, 40x) shows HE stain of rectal metastasis; panel C (lower, 20x) shows IHC of TTF-1 stain of rectal metastasis; panels D and E show lung metastasis (upper), peritoneum (middle) and rectal (lower) as evaluated by CT before crizotinib and after crizotinib, respectively; red arrows show the evaluable metastasis. IHC, immunohistochemistry; NGS, next generation sequencing; ARMS, amplification refractory mutation system; SBRT, stereotactic body radiation therapy. 
(Figure 1B). Crizotinib (250 mg), administered orally twicedaily, was added to this patient's regimen, and the PR was obtained for 12 months (Figure 1D,E). After progression via brain metastasis, the patient was received treatment with brigatinib without brain metastasis radiotherapy and the PR was obtained for 7 months. Next, she died due to progression to the brain. Total survival time was 50 months (Figure 1A).

\section{Patient 2}

A 67-year-old non-smoking male presented with widely metastatic (M1c) adenocarcinoma of the lung and bone. A CT-guided biopsy of the right lung nodule was performed and showed lung adenocarcinoma (Figure 2). The genotypes were detected using ARMS and showed EGFR to be positive and $A L K$ to be negative (Figure $2 B$ ). Therefore, treatment with pemetrexed plus cisplatin was initiated, and the PR was obtained for 7 months (Figure 2A). With the new gastrin metastasis, as confirmed by enteroscopy, the patient was diagnosed with PD (Figure $2 C$, upper panel). Re-biopsy of the gastric metastasis revealed adenocarcinoma positive for TTF-1 and CK7, while Napsin A, CDX-2, Villin and CK5/6 were negative (Figure $2 C$, middle panel). Sequencing revealed one uncommon mutation, the $M E T$ amplification (Figure 2C, lower panel). He started treatment with crizotinib, and the PR was obtained for 7 months (Figure 2D,E). However, he died in car accident.

\section{Patient 3}

A 64-year-old female with no history of smoking and a family history of lung cancer in her mother and maternal grandmother presented with widely metastatic adenocarcinoma of the lung. A CT-guided biopsy of the right lung nodule was performed, and it showed lung adenocarcinoma (Figure 3). ARMS sequencing showed $E G F R$ to be positive and $A L K$ to be negative (Figure $3 B$ ). Therefore, treatment with pemetrexed plus cisplatin was initiated, and the PR was obtained for 13 months (Figure 3A). The patient was diagnosed with PD (Figure 3C, upper panel), since new metastasis to the gastroduodenal junction was confirmed via enteroscopy. Re-biopsy of the new metastasis revealed adenocarcinoma positive for TTF-1 and CK7, while Napsin A, CDX-2, Villin and CK5/6 were negative (Figure 3C, middle panel). Sequencing revealed an EGFR exon21L858R mutation (Figure 2B). He started treatment with icotinib, and the PR was obtained for 8 months (Figure 3D,E). Next, the patient was diagnosed with PD because the tumor was increasing. NGS detection of liquid biopsy showed a KRAS mutation but not the EGFR exon 21L858R mutation. She received second-line chemotherapy and died 4 months later.

\section{Discussion}

Rossi recommended that TTF-1 and CK7 be used to assess lung cancer with GI metastasis (5). To the best of our knowledge, we are the first to report three cases of lung adenocarcinoma with metastasis to the GI tract, as confirmed using re-biopsy and IHC, one rectal, one gastrin and one at the gastroduodenal junction. There was little data to confirm the mechanism of the lung cancer with GI metastasis. This mechanism may interfere with the extracellular matrix, mesenchymal cells, tumor heterogeneity, and the coagulation system (6).

Different techniques, including liquid biopsy and tissue biopsy of NGS, are currently available to evaluate genetic alterations in cancer patients. Tumor tissue is always considered the gold standard for genotyping; however, in most cases, this material is unavailable, and liquid biopsy should be considered to be an alternative method (7). Liquid biopsy has also been used to evaluate the entire genotype of patients with wide metastasis (8). The sensitivity and specificity of tissue biopsy was $72-100 \%$ and $88-100 \%$ (9). The sensitivity and specificity of tissue biopsy was $66-100 \%$ and $80-100 \%$ (10). In comparison to tissue biopsy, several studies have shown that the consistence for tumor-specific sensitive and resistant mutations by liquid biopsy is $95 \%$ and $91 \%$, respectively $(11,12)$. First, we used NGS to compare the differences, and we found that all three patients presented with a newly occurring driver gene mutation, including ROS1 rearrangements, MET amplification and EGFR exon 21L858R, by NGS.

Recent studies and meta-analysis have demonstrated that oncogenic mutation is associated with a poor outcome due to an immune check point inhibitor (13). Therefore, the expression of PD-L1 was not performed for this patient. As is well-known, oncogenic mutations cannot appear during the disease course (14). Furthermore, we cannot confidently exclude tumor heterogeneity. However, this finding represented the newly occurring oncogenic mutations 
A Timeline and duration of each treatment

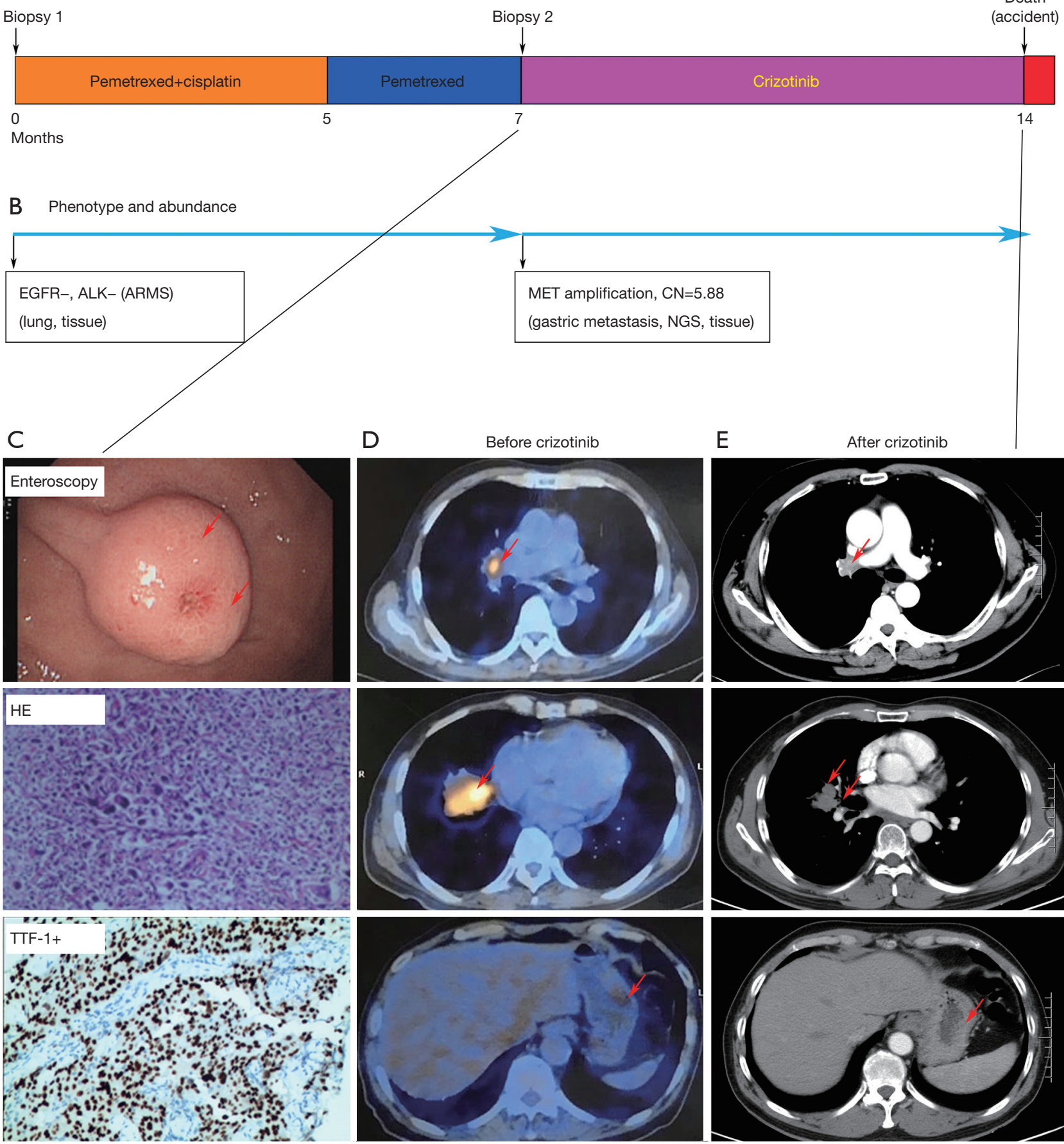

Figure 2 Lung adenocarcinoma patient with gastrointestinal metastasis benefited from re-biopsy and crizotinib. Panel A shows the various treatments of the lung adenocarcinoma patient, as well as the duration of each treatment; panel B shows the genotypes and the abundance of mutations detected by NGS for the lung adenocarcinoma patient under the various treatments; panel C (upper) shows newly occurring rectal metastasis confirmed by enteroscopy; panel $\mathrm{C}$ (middle, 20x) shows a HE stain of rectal metastasis; panel C (lower, 20x) shows IHC of a TTF-1 stain of rectal metastasis; panels D and E show the metastasis of lung (upper and middle) and gastrin (lower) as evaluated by CT before crizotinib and after crizotinib, respectively; red arrows show the evaluable metastasis. NGS, next generation sequencing; ARMS, amplification refractory mutation system; IHC, immunohistochemistry. 
A Timeline and duration of each treatment

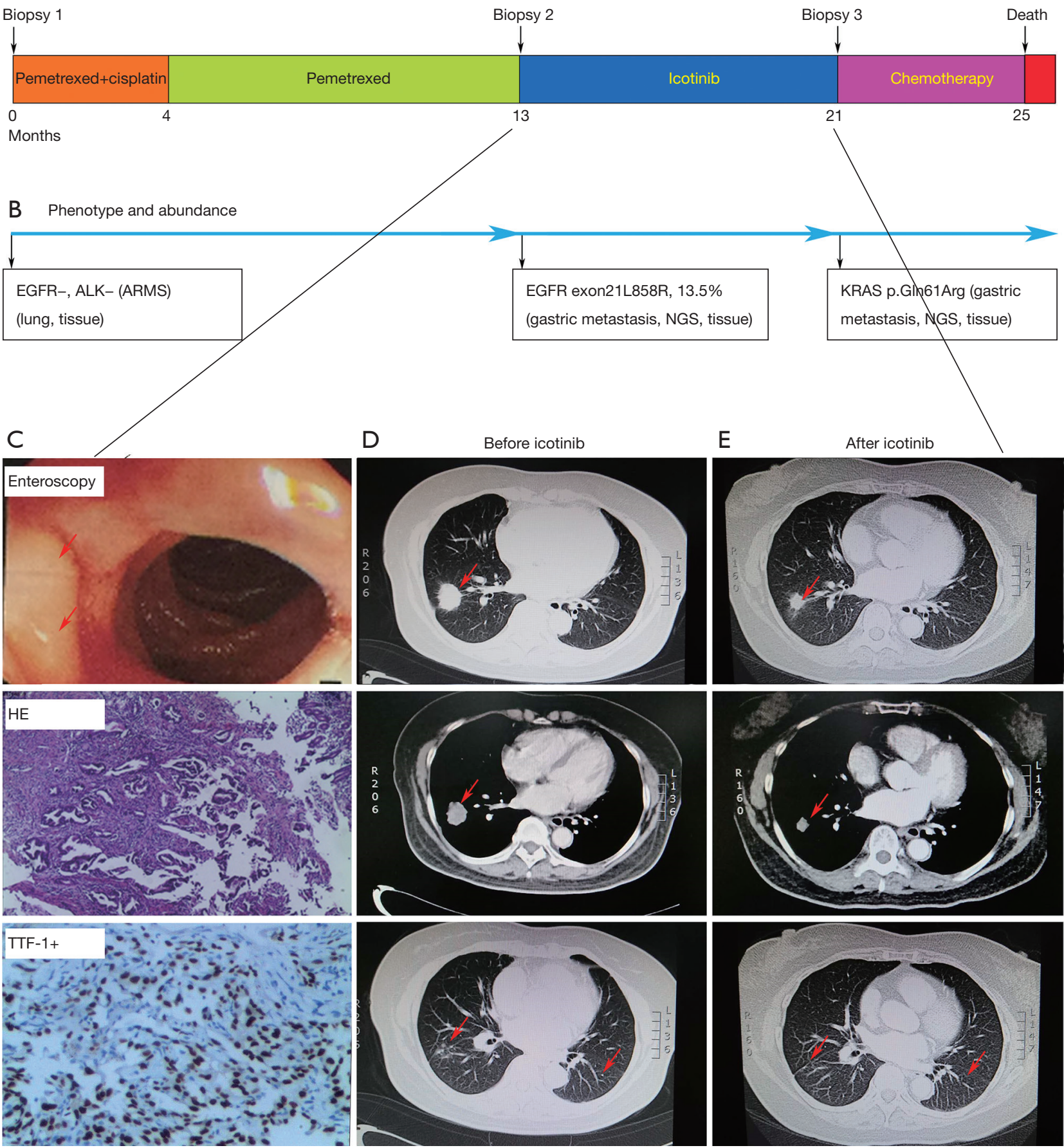

Figure 3 Lung adenocarcinoma patient progression with gastrointestinal metastasis benefited from re-biopsy and icotinib. Panel A shows the various treatments of the lung adenocarcinoma patient as well as the duration of each treatment; panel B shows the genotypes and the abundance of mutation detected by NGS for the lung adenocarcinoma patient under the various treatments; panel C (upper) shows newly occurring gastroduodenal junction metastasis, confirmed by enteroscopy; panel $\mathrm{C}$ (middle, $4 \times$ ) shows a HE stain of rectal metastasis; panel C (lower, 10x) shows IHC of a TTF-1 stain of rectal metastasis; panels D and E show the metastasis of lung evaluated by CT before icotinib and after icotinib, respectively; red arrows show the evaluable metastasis. NGS, next generation sequencing; ARMS, amplification refractory mutation system; IHC, immunohistochemistry. 
detected by high-depth NGS (1,000x for tissue and 10,000x for plasma) involved in the progression with GI metastasis.

Previous studies have shown that lung cancer with GI metastasis has a poor prognosis with mean survival of only several weeks (4). Although several findings have demonstrated the efficacy and safety of tyrosine kinase inhibitors (TKIs) in primary lung cancer with driver gene mutations in such genes as EGFR (15), ROS1 (16) and MET $(17,18)$ amplification, we still have little data on the efficacy and safety of TKIs in lung cancer progression with GI metastasis. Meanwhile, our study showed patients benefited considerably with the target therapy of newly occurring drive gene mutations. Therefore, these results suggested re-biopsy and large panel of gene detection should be performed to provide evidence for precision medicine management strategies.

\section{Acknowledgements}

Funding: This work was partially supported by the National Natural Science Foundation of China (No. 81401902 and No. 81501992) and the Hunan Natural Science Foundation (2017SK2134 and 2018JJ2238).

\section{Footnote}

Conflicts of Interest: The authors have no conflicts of interest to declare.

Informed Consent: Written informed consent was obtained from the patients for publication of this manuscript and any accompanying images.

\section{References}

1. el-Ebiary M, Torres A, González J, et al. Use of elastin fibre detection in the diagnosis of ventilator associated pneumonia. Thorax 1995;50:14-7.

2. Yoshimoto A, Kasahara K, Kawashima A. Gastrointestinal metastases from primary lung cancer. Eur J Cancer 2006;42:3157-60.

3. Joyce JA, Pollard JW. Microenvironmental regulation of metastasis. Nat Rev Cancer 2009;9:239-52.

4. Mehta RS, Liman AD, Passero VA, et al. Lung cancer with gastrointestinal metastasis - review of theories of metastasis with three rare case descriptions. Cancer Microenviron
2013;6:203-11.

5. Rossi G, Marchioni A, Romagnani E, et al. Primary lung cancer presenting with gastrointestinal tract involvement: clinicopathologic and immunohistochemical features in a series of 18 consecutive cases. J Thorac Oncol 2007;2:115-20.

6. Thiery JP, Acloque H, Huang RY, et al. Epithelialmesenchymal transitions in development and disease. Cell 2009;139:871-90.

7. Wan JCM, Massie C, Garcia-Corbacho J, et al. Liquid biopsies come of age: towards implementation of circulating tumour DNA. Nat Rev Cancer 2017;17:223-38.

8. Jung A, Kirchner T. Liquid Biopsy in Tumor Genetic Diagnosis. Dtsch Arztebl Int 2018;115:169-74.

9. Chabon JJ, Simmons AD, Lovejoy AF, et al. Circulating tumour DNA profiling reveals heterogeneity of EGFR inhibitor resistance mechanisms in lung cancer patients. Nat Commun 2016;7:11815.

10. Xu T, Kang X, You X, et al. Cross-Platform Comparison of Four Leading Technologies for Detecting EGFR Mutations in Circulating Tumor DNA from Non-Small Cell Lung Carcinoma Patient Plasma. Theranostics 2017;7:1437-46.

11. Karlovich C, Goldman JW, Sun JM, et al. Assessment of EGFR Mutation Status in Matched Plasma and Tumor Tissue of NSCLC Patients from a Phase I Study of Rociletinib (CO-1686). Clin Cancer Res 2016;22:2386-95.

12. Thress KS, Brant R, Carr TH, et al. EGFR mutation detection in ctDNA from NSCLC patient plasma: A crossplatform comparison of leading technologies to support the clinical development of AZD9291. Lung Cancer 2015;90:509-15.

13. Kobayashi K, Nakachi I, Naoki K, et al. Real-world Efficacy and Safety of Nivolumab for Advanced NonSmall-cell Lung Cancer: A Retrospective Multicenter Analysis. Clin Lung Cancer 2018;19:e349-58.

14. Bai H, Wang Z, Chen K, et al. Influence of chemotherapy on EGFR mutation status among patients with non-smallcell lung cancer. J Clin Oncol 2012;30:3077-83.

15. Mok TS, Wu YL, Thongprasert S, et al. Gefitinib or Carboplatin-Paclitaxel in Pulmonary Adenocarcinoma. N Engl J Med 2009;361:947-57.

16. Shaw AT, Ou SH, Bang YJ, et al. Crizotinib in ROS1rearranged non-small-cell lung cancer. $\mathrm{N}$ Engl J Med 2014;371:1963-71.

17. Onozato R, Kosaka T, Kuwano H, et al. Activation 
of MET by gene amplification or by splice mutations deleting the juxtamembrane domain in primary resected lung cancers. J Thorac Oncol 2009;4:5-11.

18. Liu X, Jia Y, Stoopler MB, et al. Next-Generation

Cite this article as: Zhou C, Yan B, Zeng L, Xiong Y, Liu L, Yang N, Zhang Y. Lung adenocarcinoma patient progression with gastrointestinal metastasis response to subsequent tyrosine kinase inhibitors (TKIs) from re-biopsy of new occurring driver gene mutation. J Thorac Dis 2018;10(8):E605-E611. doi: 10.21037/jtd.2018.07.97
Sequencing of Pulmonary Sarcomatoid Carcinoma Reveals High Frequency of Actionable MET Gene Mutations. J Clin Oncol 2016;34:794-802. 